\title{
The measurement of the diameter change of a piezoelectric transducer cylinder with the white-light interferometry
}

\author{
Abdelaziz Saad ${ }^{\mathrm{a}, *}$, Yi Jiang ${ }^{\mathrm{a}}$, Yuewu Liu ${ }^{\mathrm{b}}$, Zhen Wang ${ }^{\mathrm{a}}$ \\ a School of Opto-Electronics, Beijing Institute of Technology, Beijing 100081, China \\ ${ }^{\mathrm{b}}$ Key Laboratory for Mechanics in Fluid Solid Coupling Systems, Institute of Mechanics, Chinese Academy of Science, Beijing 100190, China
}

\section{A R T I C L E I N F O}

\section{Article history:}

Received 5 October 2013

Received in revised form

3 January 2014

Accepted 3 January 2014

Available online 22 January 2014

Keywords:

Piezoelectric transducer

Mach-Zehnder interferometer

White light interferometry

Fourier transform

Optical path difference

\begin{abstract}
A B S T R A C T
The measurement of the diameter change of a piezoelectric transducer (PZT) cylinder with the whitelight interferometry is proposed and experimentally demonstrated. One arm of a Mach-Zehnder interferometer (MZI) is wrapped on the PZT cylinder, and the phase change of the interferogram of the MZI is used to determine the diameter change when a DC voltage is applied on the PZT cylinder. The Fourier transform white-light interferometry is used for recovering the phase change of the interferometer. The experimental results show that the diameter change resolution of $0.8 \mathrm{~nm}$ for the PZT cylinder with diameter of $40 \mathrm{~mm}$ is achieved.
\end{abstract}

(c) 2014 Elsevier Ltd. All rights reserved.

\section{Introduction}

Piezoelectric materials have a natural property which is the ability of interaction between the electrical and mechanical states. They have attracted much attention due to their wide applications in sensing and actuating systems. Diverse styles of piezoelectric transducers have been developed with different structures such plate type, rod type, ring type, and cylinder type.

Piezoelectric cylinders are simple in construction than flat types and can easily deflect in all directions. They offer flexibility to operate under harsh condition such as high vacuum and high temperature. They are used in a wide range of applications such as atomic force microscopy (AFM) [1,2], scanning tunneling microscopes (STM) [3,4], optical fiber switch [5,6], optical fiber modulators [7], voltage sensors [8], Gyroscope [9], ultrasonic applications [10] and in ink jet printers [11].

Different methods are available for measuring piezoelectric transducer (PZT) coefficients: direct measurements of stress-induced charge [12], laser interferometers [13], laser scanning vibrometers [14], and piezoelectric force microscopes, etc. [15]. Laser interferometry methods are based on measuring the displacement deflection of the sample after an applied voltage. They have been established as popular and reliable technique for the measurement of piezoelectric coefficients $d_{31}$ and $d_{33}$ [16]. The displacement can be measured by a single-beam laser interferometer [17], where a Michelson

\footnotetext{
* Corresponding author.

E-mail address: saadabd@live.com (A. Saad).
}

interferometer is used .The reference arm length of the interferometer will change due to the piezoelectric deflection. This configuration has difficulty in separating the movement of the substrate from the dilatation of the sample. Double-beam interferometer was used to suppress the influence of the substrate movement $[18,19]$. To improve the measurement reliability and accuracy, other methods were proposed, such as scanning-modulated interferometer [20], and a Mach-Zehnder type heterodyne interferometer [21]. The crucial condition in these methods is the high resolution of an interferometer, which should be in the range of nanometer units [22]. Since the expansion of the PZT is small, the measurement is very sensitive to any small vibrations. Hence these techniques usually require a very quiet operation environment [23].

The white-light interferometry (WLI) is an attractive technique that is used for absolute measurements [24] and the measurement of the shape of objects [25], it allows the increase in the system operating range to overcome the fundamental problem that arises in conventional laser interferometry [26], and high resolution and dynamic range may be achieved [27].

In this paper, we present a technique for the PZT cylinder measurement by using the WLI. A PZT cylinder is wrapped by an arm of a MZI. We obtain the white-light interferogram when a DC voltage is applied between the faces of a PZT. The Fourier transform WLI is used for recovering the optical path difference (OPD) of the MZI [28]. Experimental measurements for diameter changes of a PZT cylinder at different driving voltages have been carried out, and experimental results show that the technique possesses high accuracy and high reliability. 


\section{Operation principle}

The experimental setup for measuring the diameter change of the PZT cylinder is shown in Fig. 1. The light source is an amplified spontaneous emission (ASE) source with the wavelengths covering $1525-1565 \mathrm{~nm}$, and the output power of $20 \mathrm{~mW}$. Two fiber optic $3 \mathrm{~dB}$ couplers are used for splitting and recombining the light. The broad-band ASE light is divided into two equal power signals through the first coupler, one goes to the reference arm, and another one goes to the sensing arm. The reference light and the sensing light recombine at the second coupler.

The sensing arm is circled on a PZT cylinder P-81 (from Heng Sheng Acoustics Electron Apparatus Company) with the size of $\phi 40 \times \phi 35 \times 30 \mathrm{~mm}$ for outside diameter, inner diameter and height, respectively. A photograph of the PZT is shown in Fig. 2. A regulated DC source (NY1303A) is used with a voltage range of $0-30 \mathrm{~V}$ and a voltage resolution of $1 \%$ to drive the PZT cylinder.

The output of the MZI is interrogated by using a CCD based optical spectrum analyzer (OSA) (BaySpec FBGA-F-1525-1565) with the resolution of $0.1 \mathrm{~nm}$. The OPD of the MZI must be smaller than the coherence length determined by the resolution of the OSA, otherwise we cannot detect the interferogram. The resolution of $0.1 \mathrm{~nm}$ respects to a coherence length of $24 \mathrm{~mm}$ at the wavelength of $1.55 \mu \mathrm{m}$. Thus the length different of the MZI between two arms must be shorter than $16.4 \mathrm{~mm}$.

In the experiment, a DC voltage is applied between the faces of a PZT cylinder. By using the Fourier transform white light interferometry [28], the OPD of the MZI can be calculated. The recovery of the OPD starts from the measurement of the phase angle $\phi . \phi$ is

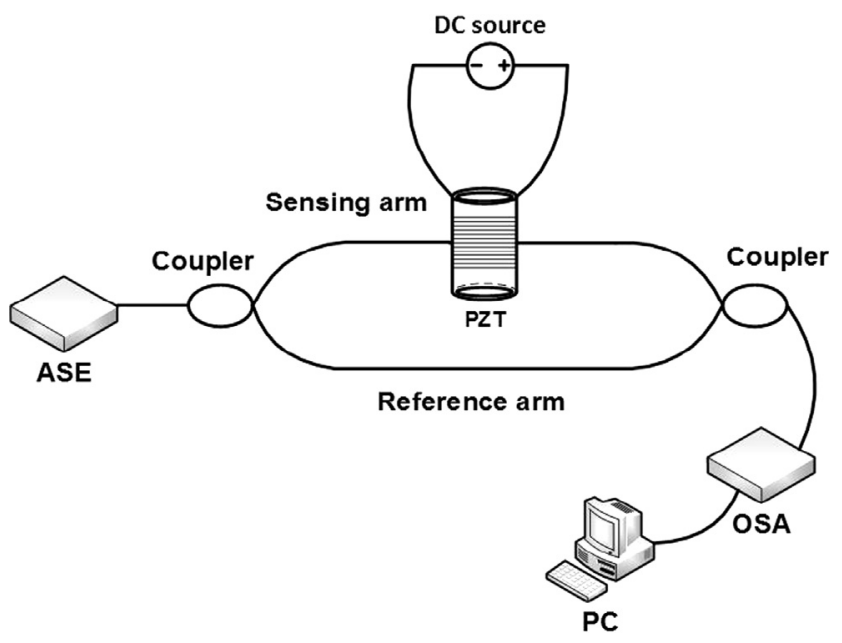

Fig. 1. Schematic diagram of the experimental setup.

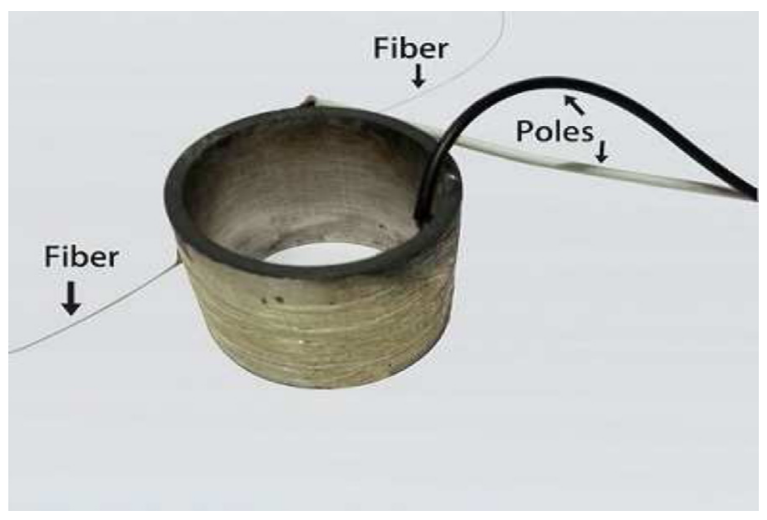

Fig. 2. Photograph of the piezoelectric transducer cylinder. given by [29]

$\phi=\frac{2 \pi}{\lambda} \cdot O P D=\frac{2 \pi}{\lambda} \cdot n \cdot L$

where $n$ is the refractive index of the fiber, and $L$ is the difference in path length between the two beams of the interferometer.

The OPD can be calculated when the phase is obtained by using Fourier transform WLI. However, when we scan the wavelength from $\lambda_{1}$ to $\lambda_{2}$, we obtain a phase change. So the OPD is obtained from the following equation:

$O P D=\frac{\left(\lambda_{1} \cdot \lambda_{2}\right)}{2 \pi \cdot\left(\lambda_{2}-\lambda_{1}\right)} \cdot \Delta \phi$

Thus, the diameter change of the PZT can be calculated from the following equation:

$\Delta D=\frac{\Delta O P D}{\pi \cdot k}$

where $\triangle O P D$ is the change of the OPD due to an applied voltage, $k$ is the number of fiber turns wrapped the PZT.

Therefore, the strain coefficient $d_{31}$ can be estimated by the following equation [30]:

$d_{31}=\frac{\Delta D \cdot t}{I D \cdot V}$

where $\Delta D$ is the diameter change of the PZT, ID is the internal cylinder diameter, $V$ is the applied voltage, and $t$ is the wall thickness.

\section{Experiment}

In this experiment, the core and cladding diameters of the fiber are $9 \mu \mathrm{m}$ and $125 \mu \mathrm{m}$ respectively, and a fiber length of $9 \mathrm{~m}$ was used as one arm of the MZI. The PZT cylinder (P-81) with strain coefficient $d_{31}=98 \mathrm{pm} / \mathrm{V}$ and an external diameter of $40 \mathrm{~mm}$ was wrapped by $5 \mathrm{~m}$ of fiber and the number of fiber turns is 40 turns. In order to protect the measured sample against vibrations, a quiet operational environment is needed.

When the wavelength is scanned from $1525 \mathrm{~nm}$ to $1560 \mathrm{~nm}$, the interference spectrum can be observed at the OSA by adjusting the OPD to be smaller than the coherence length determined by the resolution of the OSA. The optical spectrum of the MZI with an applied voltage of $0 \mathrm{~V}$ is shown in Fig. 3.

When the voltage is applied on the PZT cylinder, the interference fringes suffer a phase shift. It can be seen that the optical spectrum is red-shifted to a higher wavelength region, as shown in Fig. 4.

The OPD can be calculated by using Fourier transform WLI. Firstly the optical spectrum is Fourier transformed. The main frequency component is filtered and inverse Fourier transformed. Then we calculate a complex logarithm of the product. The phase

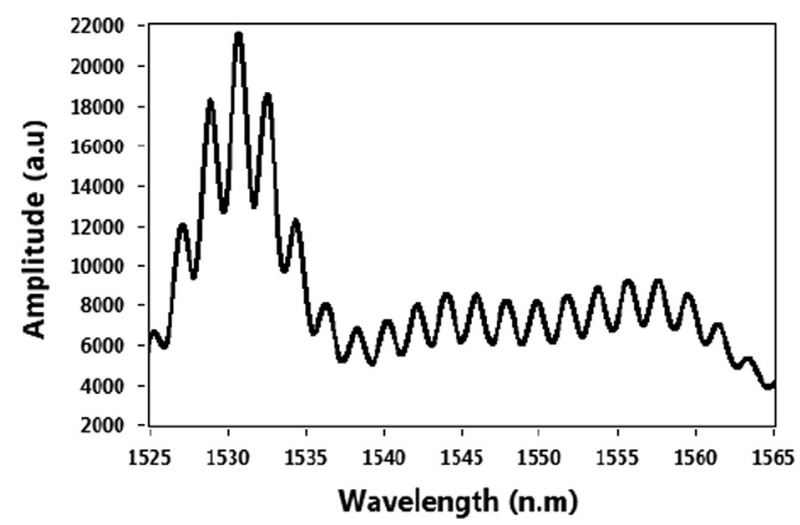

Fig. 3. The optical spectrum at $0 \mathrm{~V}$. 

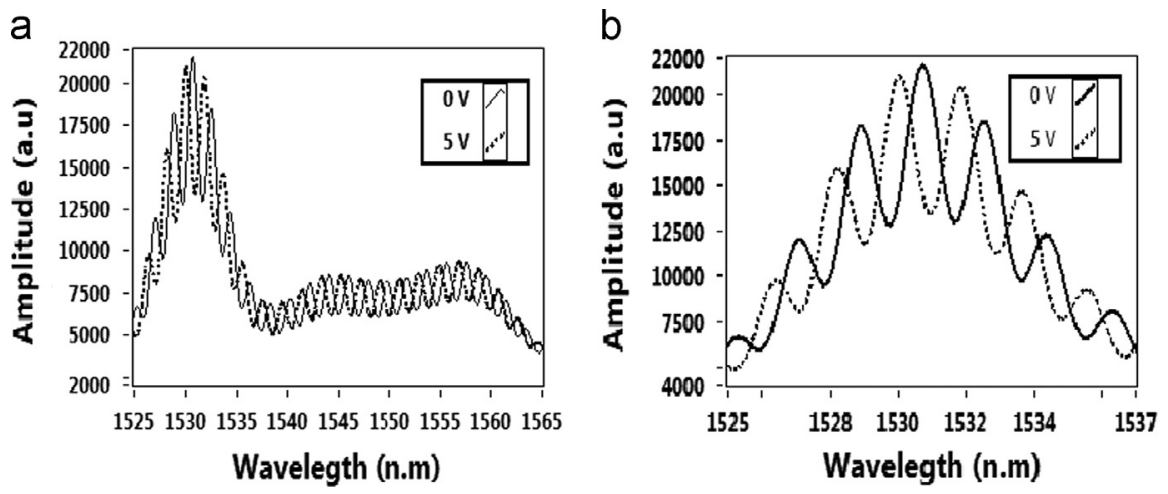

Fig. 4. (a) The optical spectrum at $0 \mathrm{~V}$ and $5 \mathrm{~V}$ and (b) local portion of the data in (a).

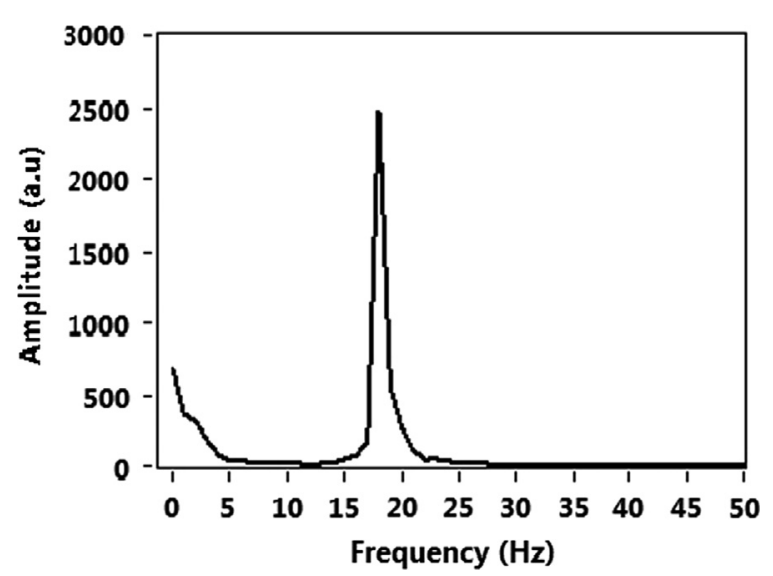

Fig. 5. The Fourier spectrum of the optical spectrum.

change of the fringe signal, which is caused by scanning wavelength, can be obtained from the imaginary part of the complex logarithm. Thus, the absolute OPD can be calculated by using the phase change [31].

The Fourier spectrum is shown in Fig. 5. The spectrum was filtered and inverse Fourier transformed, as shown in Fig. 6. The interferometric phase changes is shown in Fig. 7. When the wavelength is scanned from $1525 \mathrm{~nm}$ to $1560 \mathrm{~nm}$, the phase change is $113.09 \mathrm{rad}$ and the OPD is calculated to be $1241.21 \mu \mathrm{m}$ for an applied voltage of $0 \mathrm{~V}$.

For an applied voltage of $25 \mathrm{~V}$, the measured OPD is $1245.68 \mu \mathrm{m}$. Therefore, the change of the OPD can be calculated. Using Eqs. (3) and (4), the diameter change of the PZT cylinder and the strain coefficient $d_{31}$ are $0.035 \mu \mathrm{m}$ and $100 \mathrm{pm} / \mathrm{V}$, respectively, which is agreed to the strain coefficient provided by the manufacturer of the PZT.

Fig. 8 shows the measured OPD change for various applied voltages from $0 \mathrm{~V}$ to $25 \mathrm{~V}$ by an increment step of $5 \mathrm{~V}$. A hysteresis cycle can be observed, the maximum and minimum OPD changes were found to be $1245.68 \mu \mathrm{m}$ and $1241.21 \mu \mathrm{m}$ at $25 \mathrm{~V}$ and $0 \mathrm{~V}$, respectively. It can be seen that the value of the OPD decreases significantly from their value at high DC bias after the DC bias is removed.

In order to test the stability of the system, we measured the OPD 30 times at an applied voltage of $29 \mathrm{~V}$. The measured results are distributed between 1246.663 and $1246.86 \mu \mathrm{m}$, as shown in Fig. 9. The fluctuation of the measurement results is $0.1 \mu \mathrm{m}$ which shows that the stability of the system is well. For a PZT cylinder wrapped by a fiber length of $5 \mathrm{~m}$, the resolution for the measurement of diameter change of the PZT cylinder that can be achieved is $0.8 \mathrm{~nm}$. In fact; the stability can be significantly increased by doing an average calculation, to reduce any environmental effect.

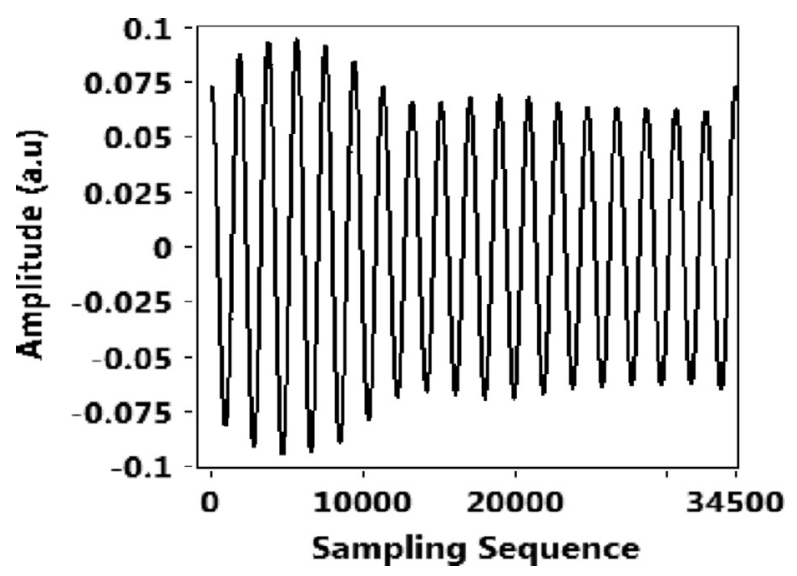

Fig. 6. Signal filtered, and inverse transformed.

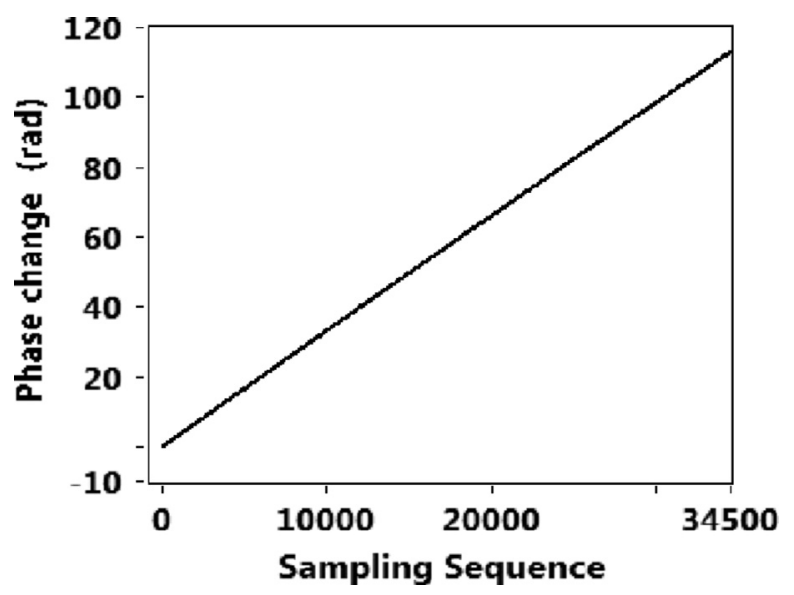

Fig. 7. Phase changes for an applied voltage of $0 \mathrm{~V}$.

The main reasons of errors in the measurement of diameter change are the resolution of the optical spectrum analyzer and the sensitivity to any external noises and environmental fluctuations.

\section{Conclusion}

In conclusion, we have presented a white-light interferometric method for the measurement of the diameter change of a PZT cylinder. The output of the MZI is interrogated by using an OSA, and the phase change of the interference spectrum during the applied voltage was used for the determination of the PZT diameter change. 


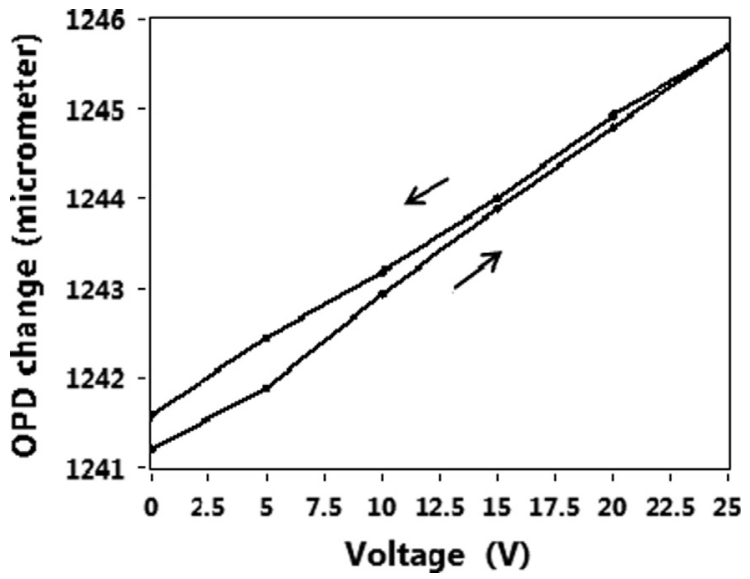

Fig. 8. Measured OPD change for various applied voltages.

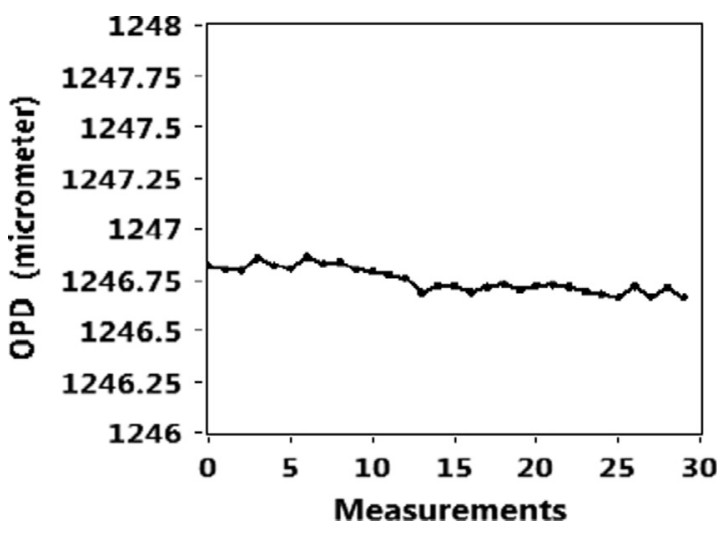

Fig. 9. Results of continuous tests at $29 \mathrm{~V}$.

The proposed method possesses the abilities to provide wide dynamic measurement range, low cost without other additional components, and offers advantage of easier fabrication. However, it requires a very quiet working environment. A resolution of up to $0.8 \mathrm{~nm}$ is achieved in our experiment. Thus the technique appears to have potential applications such as optical phase modulator, fiber stretching, and deformation measurement.

\section{Acknowledgments}

This work was supported by Natural Scientific Foundation of China (51075037), National Sci and Tech Key Project of China (2011ZX05038003), and the Doctoral Foundation of Education Ministry of China (20101101110014).

\section{References}

[1] Abramovitch DY, Andersson SB, Pao LY, Schitter G. A tutorial on the mechanisms, dynamics, and control of atomic force microscopes. In: American control conference, 2007 (ACC'07). IEEE; 2007. p. 3488-502.

[2] Kuiper S, Schitter G. Active damping of a piezoelectric tube scanner using selfsensing piezo actuation. Mechatronics 2010;20(6):656-65.

[3] Gawthrop P, Bhikkaji B, Moheimani S. Physical-model-based control of a piezoelectric tube for nano-scale positioning applications. Mechatronics 2010;20(1):74-84.
[4] Carr R. Finite element analysis of PZT tube scanner motion for scanning tunnelling microscopy. J Microsc 1988;152(2):379-85.

[5] Leung M, Yue J, Razak K, Haermnerle E, Hodgson M, Gao W. Development of a $1 \times 2$ piezoelectric optical fiber switch. In: Proceedings of SPIE, the international society for optical engineering. Society of Photo-Optical Instrumentation Engineers; 2008, p. 683603-1.

[6] Ueda M, Sawada H, Tanaka A, Wakatsuki N. Piezoelectric actuator using a linbo 3 bimorph for an optical switch. In: Proceedings of the IEEE 1990 ultrasonics symposium. IEEE; 1990. p. 1183-6.

[7] Jin GC, Bao NK. Surface detection and 3d profilometry for microstructure using optical metrology. Opt Lasers Eng 2001;36(1):1-9.

[8] Laskovski AN, Yuce MR, Moheimani SR. Fm-based piezoelectric strain voltage sensor at ultra-low frequencies with wireless capability. Sensors Actuators A: Phys 2013;199:49-55.

[9] Yang J, Fang H. A new ceramic tube piezoelectric gyroscope. Sensors Actuators A: Phys 2003;107(1):42-9.

[10] Zhang H, Zhang Sy, Fan L. Simplified formulae to investigate flexural vibration characteristics of piezoelectric tubes in ultrasonic micro-actuators. Ultrasonics 2010;50(3):397-402.

[11] Chen W, Tsao S. Ink jet head. IBM Tech Discl Bull 1977;20(2):504-5.

[12] Barzegar A, Damjanovic D, Setter N. Analytical modeling of the apparent d/sub 33/piezoelectric coefficient determined by the direct quasistatic method for different boundary conditions. IEEE Trans Ultrason Ferroelectr Freq Control 2005;52(11):1897-903.

[13] Tsurumi T, Ikeda N, Ohashi N. Non-180 domain contribution in electric-fieldinduce strains of PZT ceramics measured by a Mach-Zehnder interferometer. Nippon Seramikkusu Kyokai Gakujutsu Ronbunshi 1998;106(11):1062-6.

[14] Yao K, Tay FEH. Measurement of longitudinal piezoelectric coefficient of thin films by a laser-scanning vibrometer. IEEE Trans Ultrason Ferroelectr Freq Control 2003:50(2):113-6.

[15] Huang Z, Zhang Q, Corkovic S, Dorey R, Whatmore RW. Comparative measurements of piezoelectric coefficient of PZT films by berlincourt, interferometer, and vibrometer methods. IEEE Trans Ultrason Ferroelectr Freq Control 2006;53(12):2287-93.

[16] Burianova L, Šulc M, Prokopova M. Determination of the piezoelectric coefficients of PZT ceramics and composites by laser interferometry. J Eur Ceram Soc 2001;21(10):1387-90.

[17] Zhang Q Pan W, Cross LE. Laser interferometer for the study of piezoelectric and electrostrictive strains. J Appl Phys 1988;63(8):2492-6.

[18] Kholkin A, Wutchrich C, Taylor D, Setter N. Interferometric measurements of electric field-induced displacements in piezoelectric thin films. Rev Sci Instrum 1996;67(5):1935-41.

[19] Pan W, Cross L. A sensitive double beam laser interferometer for studying high-frequency piezoelectric and electrostrictive strains. Rev Sci Instrum 1989:60(8):2701-5.

[20] Wang Z, Zhu W, Miao J, Chao C, Tan OK. Measurement of longitudina piezoelectric coefficient of film with scanning-modulated interferometer. Sensors and Actuators A: Phys 2006;128(2):327-32.

[21] Lueng C, Chan H, Surya C, Fong W, Choy C, Chow P, et al. Piezoelectric coefficient of GAN measured by laser interferometry. J Non-Cryst Solids 1999:254(1):123-7.

[22] Fialka J, Benes P. Comparison of methods of piezoelectric coefficient measurement. In: IEEE international on instrumentation and measurement technology conference (I2MTC). IEEE; 2012. p. 37-42.

[23] Huang Z, Whatmore R. A double-beam common path laser interferometer for the measurement of electric field-induced strains of piezoelectric thin films. Rev Sci Instrum 2005:76(12):123906.

[24] Jiang Y, Ding W, Liang P, Fu L, Wang C. Phase-shifted white-light interferometry for the absolute measurement of fiber optic Mach-Zehnder interferometers. J Lightwave Technol 2010;28(22):3294-9.

[25] Pavliček P, Michálek V. White-light interferometry envelope detection by Hilbert transform and influence of noise. Opt Lasers Eng 2012;50(8):1063-8.

[26] Kumar UP, Haifeng W, Mohan NK, Kothiyal M. White light interferometry for surface profiling with a colour ccd. Opt Lasers Eng 2012;50(8):1084-8.

[27] Yuan L. White light interferometric fiber optic sensors: for structural monitoring. LAP Lambert Acad. Publ.; 2010 ISBN 9783838345826.

[28] Jiang Y. Fourier transform white-light interferometry for the measurement of fiber-optic extrinsic Fabry-Perot interferometric sensors. IEEE Photon Technol Lett 2008;20(2):75-7.

[29] Yu B. Development of tunable optical filters for interrogation of white-light interferometric sensors [Ph.D. thesis]. Virginia Polytechnic Institute and State University; 2005.

[30] Ceramic P. Piezo technology; 2012.

[31] Jiang Y, Ding W. Recent developments in fiber optic spectral white-light interferometry. Photon Sensors 2011;1(1):62-71. 